\title{
ANÁLISIS PRELIMINAR DE DULCE DE PAPA AMARILLA (Solanum goniocalyx)
}

Dániza M. Guerrero Alva'

\author{
R E S U M E N
}

\begin{abstract}
El manjar de papa amarilla es un preparado tradicional en el Perú. En el presente estudio se caracterizó la materia prima: papa amarilla (Solanum goniocalyx) y azucar. La composición promedio de la leche evaporada es reportada por la bibliografía correspondiente.
\end{abstract}

Seguidamente, se determinó el flujo básico de procesamiento, parámetros y rendimiento, asi como las relaciones que permiten deducir una formulación teórica.

La variación del flujo artesanal (calentamiento de la pre mezcla papa) leche hasta $56^{\circ} \mathrm{C}$, e incorporación de azúcar) produjo un dulce de mejores caracteristicas y mayor rendimiento en menor tiempo de procesamiento y gasto de combustible. Asimismo, mediante una prueba sensorial se observó que no era aceptada la sustitución de leche evaporada por leche en polvo, en ninguno de los porcentajes ensayados. Algunos defectos que pueden presentarse en el producto final se tratan relacionados con la causa probable de origen finalmente, se tua determinado la composición proximal promedio asi como sniestabilidad mecraliológica (almacenaje a $12^{\circ} \mathrm{C}$ por 60 dias).

\section{INTRODUCCIÓN}

\section{DEFINICIÓN DE DULCE DE LECHE}

El dulce de leche o «manjarblanco» como se le conoce específicamente en nuestro pais, ha sido definido por IRAM (1971) como el producto alimenticio obtenido por calentamiento y concentración a $100^{\circ} \mathrm{C}$ de temperatura y presión atmosférica, de la mezcla constituida por leche entera, sacarosa y otros azúcares diferentes, y que se caracteriza por su sabor dulce y agradable, color caramelo clavo, olor delicado y consistencia firme y uniforme, pudiendo ser fácilmente untable. De alli se explica la amplia aplicación de uso que tiene como relleno y cobertura en la industria pastelera.

Este dulce tan conocido y apreciado por sus caracteristicas, en el Perú y otros países del continente, empezó a prepararse familiarmente en

\section{Ingeniero en Industrias Alimentarias}

- Colaboraron como asistentes: Maria Huertas, L. Valenzuela, N. Hernández, P. Burga, A. Mendiola, V. Huarcaya y H. Montes.

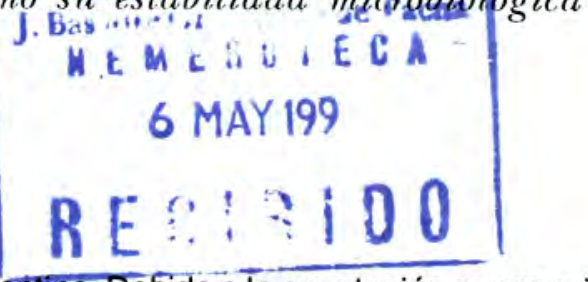

Argentina. Debido a Ta aceptación conseguida en 1907 se inició en ese país la producción industrial del dulce de leche. Hasta la fecha, tanto el proceso como los insumos han ido innovándose progresivamente. Así puede trabajarse en proceso cerrado y baja presión y se permite la adición de estabilizantes, saborizantes y aromatizantes, que han dado lugar a denominaciones y tipos de dulces de leche aplicables solo a los productos que se ajustan a la definición anterior, con el añadido de otros insumos como los siguientes:

\footnotetext{
1. Dulce de leche semidescremado: si se procesa con leche semidescremada

2. Dulce de leche con chocolate : al que se añade no más de $1,5 \%$ de cacao.

3. Dulce de leche con mani o agregados que deben figurar en almendra $\%$ en el rotulado.

4. Dulce de leche con almidón: : Que incorpora $0,5 \%$ de almidón como máximo.
}

Fuente: FREYER, Julio 1972 (citado por CHACON, Hugo 1978). 
. Por otro lado, la composición final del dulce de leche es bastante variable pero puede ser resumida de acuerdo a la información recopilada por Chacón, de la siguiente manera:

COMPOSICIÓN PROXIMAL DEL DULCE DE LECHE

\begin{tabular}{|l|l|l|}
\hline CONSTITUYENTE & PROMEDIO(\%) & $\begin{array}{c}\text { RANGO DE } \\
\text { VARIABILIDAD(\%) }\end{array}$ \\
\hline Humedad & 20 & \pm 5 \\
Sacarosa & 42,5 & $\pm 5,5$ \\
Sólidos de leche & 28 & \pm 2 \\
Materia Grasa & 6 & \pm 4 \\
Proteina & 7 & \pm 1 \\
Lactosa & 12,5 & \pm 2.5 \\
Ceniza & 1,5 & $\pm 0,5$ \\
Ac. Láctico & 0,2 & NO REPORTAN \\
& & DATOS \\
\hline
\end{tabular}

Pero, a pesar de las caracteristicas inherentes del producto, como son la elevada concentración de azúcares y sólidos de leche, es susceptible de presentar defectos o alteraciones en el tiempo, pudiendo ocurrir:

a) Cristalización de la sacarosa y lactosa, que se perciben en la consistencia arenosa del alimento; asi como por los cristales grandes y largos que puede formar la gràsa láctea.

b) Fermentación debida a la presencia de lévaduras, que hidrolizan la lactosa con producción de alcohol etílico, anhidrido carbónico y otras sustancias secundarias no deseadas.

c) Desarrollo de hongos y bacterias, indicadoras de falta de higiene, los cuales proliferan fácilmente cuando el contenido de humedad es alto.

d) Formación de grumos blandos y elásticos debidos a la precipitación de la caseina y por la elevada acidez de la mezcla.

e) Sinéresis, cuando el dulce de leche tiene más de $35 \%$ de humedad o la acidez es elevada.

f) Consistencia gomosa, por exceso de neutralizantes.

g) Color muy oscuro, se produce cuando el tiempo de procesamiento es muy prolongado, por la caramelización de los azúcares ó por el añadido de leche de muy baja acidez.

Para contrarrestar efectos no deseados se han probado diferentes formas de incorporar el azúcar o la leche durante el procesamiento, evaluación de la calidad quimica y sanitaria de la leche, uso de conservadores, neutralizantes y control estricto del momento en el que concluye la operación de concentración, a fin de conseguir un dulce de calidad estable, mejorar rendimientos, y de características químicas, microbiológicas y sensoriales óptimas.

En nuestro pais se ha desarrollado una formulación en base al procedimiento tradicional de fabricación de Dulce de Leche (proceso abierto y presión atmosférica), con el añadido de $2 \%$ de glucosa para evitar los problemas debidos a la cristalización de azúcares y $0,5 \%$ de almidón de maiz consiguiéndose un producto organolépticamente estable durante un periodo de almacenaje de 30 dias a temperatura ambiente (Chacón, H. 1978). El flujo de operaciones resultado de esta investigación es el siguiente:

DIAGRAMA DEL FLUJO DE PROCESAMIENTO DE DULCE DE LECHE

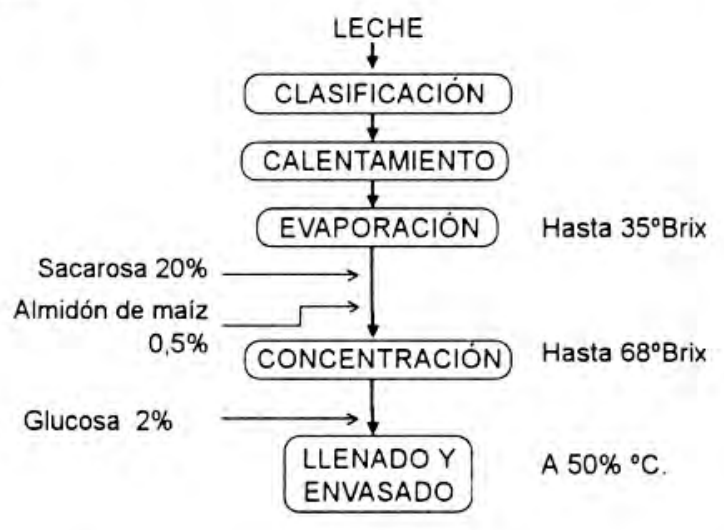

Sin embargo, el ingenio popular aunado a la diversidad de materias primas con las que cuenta el Perú, ha creado un dulce en el que se sustituye de manera significativa la leche entera por papa, preferentemente amarilla, para aprovechar de este modo su riqueza de materia seca, composición química y color amarillo intenso, que realza más aún las caracteristicas de este producto tradicional llamado "manjar de papa".

Es por ello que se ha realizado este estudio, para conocer y precisar sus características químicas, sensoriales y sanitarias; y poder mejorar el procedimiento tradicional de producción, así como evaluar la perspectiva industrial desde el punto de vista de rendimiento.

Además, este aporte, que proviene del conocimiento popular, sin duda, merece estudiarse dentro del marco del impulso que se desea dar a la producción nacional de papa amarilla, como una nueva alternativa industrial. 


\section{MATERIALES Y MÉTODOS}

\subsection{MATERIALES}

- La materia prima usada en el presente estudio fue la papa amarilla del Centro o Runtus (Solanum goniocalyx) cuyo origen es la Sierra Central del Perú (departamento de Junin), N CIP 702472. leche evaporada entera nacional y azúcar blanca industrial.

- Adicionalmente, en una de las fases del trabajo se utilizó leche en polvo entera y reconstituida.

-También se hizo uso de equipos, material de vidrio, instrumentos y utensilios apropiados para la realización de los ensayos.

\subsection{MÉTODOS}

- Antes de iniciar las pruebas, se reconoció la composición proximal y caracteristicas varietales de la papa amarilla del Centro (Solanum goniocalyx), de la leche y azúcar utilizados.

- Seguidamente se determinó la fórmula base del dulce de papa, asi como la expresión de la fórmula teórica. Con la formulación base y siguiendo el flujo tradicional de procesamiento se desarrollaron las mediciones de los parámetros fundamentales.

- Se varió el flujo artesanal probando la incorporación total de la leche al inicio del proceso productivo y del azúcar después de 10 minutos de calentamiento, con la medición de los parámetros fundamentales.

- Definición del flujo definitivo de procesamiento y descripción de las operaciones.

- Sustitución parcial de la leche evaporada por leche en polvo reconstituida. Análisis sensorial para la elección de la mejor muestra.

- Defectos que pueden producirse en el dulce de papa.

- Composición proximal de la formulación definitiva (60 dias a $12^{\circ} \mathrm{C}$ ).

\section{RESULTADOS Y DISCUSIÓN}

\subsection{CARACTERISTICAS DE LOS INSUMOS USADOS EN LA FORMULACION}

a) Papa amarilla: todos los ensayos han sido trabajados con la variedad amarilla del Centro, o Runtus (Solanum goniocalyx).

Esta variedad proviene de plantas de crecimiento semierecto, de tallo y hojas verde claro presentando estas últimas rugosidades. Las flores son abundantes al igual que sus frutos, caracterizados por su forma oval o redondeada, piel amarillo claro y pulpa de color amarillo intenso, con ojos profundos. Es muy harinosa y tiene alto contenido de materia seca $(27,50 \%)$, que, aunque difiere de los valores reportados por el Centro Internacional de la Papa (C.I.P.) para esta variedad (32 a $34 \%$ de materia seca para Solanum goniocalyx), es mayor que para la variedad blanca $(25,50 \%)$ y los resultados obtenidos por otros investigadores $(26,80 \%)$, (Collazos C. 1993). Por otro lado, el contenido de proteina $(2,18 \%)$ es interesante por ser superior al que presentan otros tubérculos y raices (camote amarillo $1,2 \%$, oca $1 \%$, olluco $1,1 \%$, pituca $1,6 \%$ o yuca amarilla $0,6 \%$ (Collazos C. 1993).

CUADRO N 01: Composición proximal de la papa amarilla. Resultados comparativos.

\begin{tabular}{|l|l|l|l|}
\hline \multirow{2}{*}{ CONSTITUYENTE } & \multicolumn{3}{|c|}{ CANTIDAD } \\
\cline { 2 - 4 } & $\left({ }^{\circ}\right)$ & $\left({ }^{\circ}\right)$ & $\left({ }^{\circ 00}\right)$ \\
\hline Humedad (\%) & 73,2 & 73,2 & 72,5 \\
Materia seca (\%) & 26,80 & 26,8 & 27,50 \\
Proteina (\%) & 2,0 & 2,0 & 2,18 \\
Grasa (\%) & 0,4 & 0,4 & 2,71 \\
Ceniza (\%) & n.r. & 1,1 & 0,82 \\
Fibra (\%) & n.r. & 0,7 & 0,179 \\
Carbigudratis (\%) & n.r. & n.r. & 21,61 \\
Gravedad especifica (gr/ml) & n.r. & n.r. & 1,449 \\
pH $\left(23,9^{\circ} \mathrm{C}\right)$ & n.r. & n.r. & 6,38 \\
\hline
\end{tabular}

$\left({ }^{\circ}\right)$ Antunez de Mayolo Santiago 1988

$\left({ }^{\circ}\right)$ Collazos Carlos, 1993

$\left({ }^{\circ 00}\right)$ Var. Amarilla del Centro, Runtus (Solanum goniocalyx, enero de 1996, usada en el experimento.

n.r. no reporta

Pero es importante mencionar que la papa amarilla también aporta vitaminas y minerales; principalmente ácido ascórbico $(9 \mathrm{mg})$, fósforo $(52 \mathrm{mg})$ y Calcio $(6 \mathrm{mg})$; y en menor proporción otros constituyentes importantes como Fierro $(0,4 \mathrm{mg})$ y niacina $(1,85 \mathrm{mg})$ de acuerdo a Antúnez de Mayolo (1988) y Collazos et tal (1993).

b) Leche: en la formulación se utilizó leche evaporada entera con $7,5 \%$ de tenor graso y $25 \%$ de sólidos totales de acuerdo a rotulado.

Se hicieron ensayos sustituyendo la leche evaporada con leche en polvo entera y reconstituida. 
La composición promedio de la leche evaporada entera investigada por Collazos (1993), así como la correspondiente a la leche en polvo entera usada se presentan en el siguiente cuadro.

CUADRO N ${ }^{\circ}$ 02: Composición promedio de la leche evaporada entera $\left({ }^{\circ}\right)$ y leche en polvo entera $\left({ }^{\circ}\right)$.

\begin{tabular}{|lc|c|c|}
\hline \multicolumn{2}{|c|}{ CONSTITUYENTE } & $\begin{array}{c}\text { LECHE } \\
\text { EVAPORADA }\end{array}$ & $\begin{array}{c}\text { LECHE } \\
\text { EN POLVO }\end{array}$ \\
\hline Agua & $(\mathrm{gr})$ & 72,4 & 2,8 \\
Proteina & $(\mathrm{gr})$ & 7,0 & 27,2 \\
Grasa & $(\mathrm{gr})$ & 8,1 & 28,5 \\
Carbohidratos & $(\mathrm{gr})$ & 10,9 & 35,9 \\
Fibra & $(\mathrm{gr})$ & -- &.-- \\
Ceniza & $(\mathrm{gr})$ & 1,6 & 5,6 \\
Calcio & $(\mathrm{mg})$ & 231 & 890 \\
Fósforo & $(\mathrm{mg})$ & -- & 620 \\
Fierro & & -- & -- \\
Retinol & $(\mathrm{mg})$ & 52 & $\ldots-$ \\
Tiamina & $(\mathrm{mg})$ & 0,03 & 0,3 \\
Riboflavina & $(\mathrm{mg})$ & 0,66 & 1,1 \\
Niacina & $(\mathrm{mg})$ & 0,31 & -- \\
Ácido ascórbico & & 000 & -- \\
\hline
\end{tabular}

(') Collazos Carlos, 1993

$\left({ }^{\circ}\right)$ Información dada en el rotulado

c) Azúcar: se trabajó con azúcar blanca nacional.

Los resultados obtenidos del análisis del azúcar blanca se presentan a continuación.

CUADRO $N^{\circ}$ 03: Composición del azúcar usado en la elaboración de dulce de papa.

\begin{tabular}{|l|l|c|}
\hline \multicolumn{1}{|c|}{ CONSTITUYENTE } & \multicolumn{1}{|c|}{ VALOR } & REFERENCIA \\
\hline Humedad & $0,048 \%$ & Máx, 0,12\% según ITINTEC \\
Cenizas & 0,03 & Máx, 0,06\% según ITINTEC \\
pH & 5,6 & \\
Densidad de carga (s/a) & $0,80252 \mathrm{gr} / \mathrm{ml}$ & \\
Color (lcumsa) & 4,8 & Máx, 10\% según PEARSON \\
Azúcares reductores & $0,0967 \%$ & Máx, 0,1\% según PEARSON \\
\hline
\end{tabular}

De acuerdo a la norma técnica nacional (ITINTEC) asi como a la bibliografía consultada se puede decir que este insumo es de muy buena calidad y cumple ampliamente con las especificaciones.

\subsection{FORMULA BASE DEL DULCE DE PAPA}

Después del trabajo de campo y los ensayos previos realizados de acuerdo a los datos empiricos recopilados, se elaboró el flujo de procesamiento del dulce de papa a pequeña escala y en sistema abierto, que se presenta a continuación.

DIAGRAMA N ${ }^{\circ} 01:$ Flujo base para el procesamiento de dulce de papa amarilla.

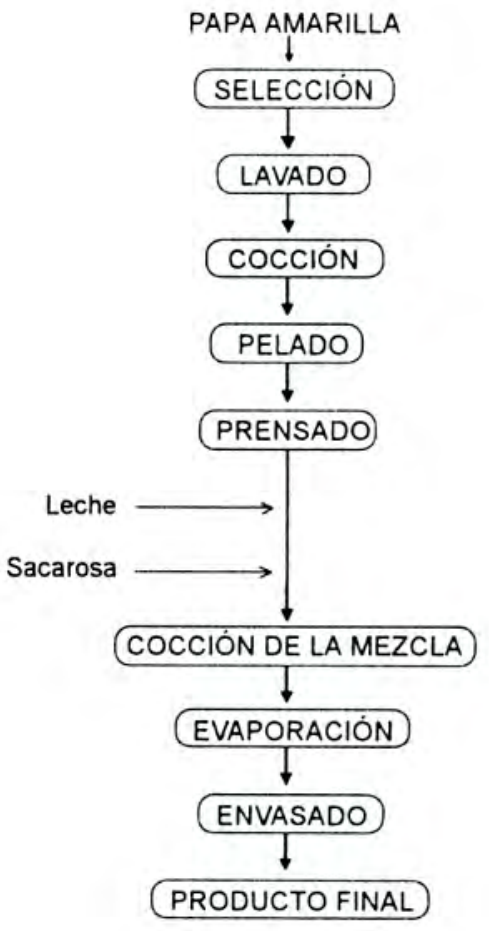

Opcionalmente en el punto final se puede saborizar con vainilla o canela en polvo.

Basándonos en la tabla de Santos (1976) (citado por Chacón H. 1978) para deducir formulaciones teóricas de dulce de leche se añadió la proporción de azúcar utilizado en la formulación asi como los constituyentes principales y correspondientes a la papa amarilla, considerando como promedio los valores dados por Collazos (1993) para esta variedad de papa y la leche evaporada entera, con los siguientes resultados: 
CUADRO N 04: Formulación teórica y básica de dulce de papa amarilla y leche evaporada entera (").

\begin{tabular}{|c|c|c|c|}
\hline CONSTITUYENTE & & $\begin{array}{l}\text { CÓDIGO O } \\
\text { FORMULA }\end{array}$ & CANTIDAD \\
\hline $\begin{array}{l}\text { Azúcar adicional a la } \\
\text { papa amarilla respecto } \\
\text { a la mezcla inicial }\end{array}$ & $(\%)$ & a & 30 \\
\hline Grasa en la leche & $(\%)$ & b & 8,1 \\
\hline Sólidos lácteos de leche & $(\%)$ & c & 27,6 \\
\hline $\begin{array}{l}\text { Grasa en la papa } \\
\text { amarilla }\end{array}$ & $(\%)$ & d & 0,4 \\
\hline Proteina en la papa & $(\%)$ & $\mathrm{e}$ & 2,0 \\
\hline $\begin{array}{l}\text { Sólidos de papa } \\
\text { amarilla }\end{array}$ & $(\mathrm{kg})$ & $f$ & 26,80 \\
\hline Sólidos totales & $(\mathrm{kg})$ & $g=a+c+f$ & 84.40 \\
\hline $\begin{array}{l}\text { Manjarblanco de papa } \\
\text { con } 60 \% \text { de sólidos }\end{array}$ & $(\mathrm{kg})$ & $h=g / 0,6$ & 141.83 \\
\hline Agua residual & $(\mathrm{kg})$ & $\mathrm{i}=\mathrm{h}-\mathrm{g}$ & 56.73 \\
\hline $\begin{array}{l}\text { Proteina en el } \\
\text { manjarblanco de papa }\end{array}$ & $(\%)$ & $j=7+e / h$ & 6.47 \\
\hline $\begin{array}{l}\text { Grasa en el manjar } \\
\text { blanco de papa }\end{array}$ & $(\%)$ & $k=b+d / h$ & 7,62 \\
\hline Lactosa en solución & $(\%)$ & $m=10,9 / i+10,9$ & 16,11 \\
\hline $\begin{array}{l}\text { Azúcar adicionado en el } \\
\text { manjarblanco de papa } \\
\text { amarilla }\end{array}$ & $(\%)$ & $\mathrm{n}=\mathrm{a} / \mathrm{h}$ & 21,15 \\
\hline
\end{tabular}

(*) Composición promedio para la papa amarilla y leche evaporada entera dadas por COLLAZOS y col., 1993.

Como se puede apreciar, la formulación teórica está en función de la composición de la papa amarilla en primera instancia y si se añade agua para facilitar el prensado de la papa. La leche evaporada puede ser estandarizada con mayor facilidad industrialmente.

Por otro lado, y de acuerdo al flujo correspondiente al diagrama $\mathrm{N}^{\circ} 01$, se hicieron mediciones de la temperatura de trabajo, la consistencia de la mezcla en términos de grado de desparramamiento (medido en centímetros para un ángulo de $\mathrm{O}^{\circ}$ ó nivel muerto), el tiempo que demora la operación, así como el color relacionado con reacciones de caramelización de azúcares y de Maillard.

El diagrama $N^{0} 02$ nos muestra la variación de la temperatura y el grado de desparramamiento (en $\mathrm{cm}$ ) durante el procesamiento. En estos gráficos podemos observar que la temperatura asciende rápidamente durante los 20 minutos iniciales de la concentración, decreciendo el gradiente por la consistencia de la mezcla. Este último efecto inicia cuando la temperatura de trabajo ha superado los $85^{\circ} \mathrm{C}$, estando cerca el final del proceso. Es importante considerar que la temperatura nunca es superior a $100^{\circ} \mathrm{C}$, para poder controlar la calidad sanitaria del dulce de papa.

De otra parte, la consistencia de la pulpa de papa se torna fluida con la incorporación de los insumos, siendo máxima en los primeros diez minutos de iniciado el proceso de concentración por evaporación para descender considerablemente hasta un valor de $7,3 \mathrm{~cm}$, correspondiente al final de la evaporación. En esta condición el dulce de papa obtenido posee $60 \%$ de sólidos, densidad de 0,113 $\mathrm{gr} / \mathrm{ml}$ a $20^{\circ} \mathrm{C}$ y un rendimiento de $61 \%$ superior al de Chacón (40\%) y González (42\%) (citado por Chacón H. 1978). Estos resultados pueden deberse a que los gránulos de almidón de papa son grandes, y a mayor tamaño empastan a menor temperatura que otros almidones (alrededor de $65^{\circ} \mathrm{C}$ como se observa en el gráfico que relaciona la temperatura y la consistencia), logrando gelatinizar a temperaturas menores de $95^{\circ} \mathrm{C}$. Los granos de almidón de papa están constituidos por amilosa y amilopectina cuya proporción influye durante la cocción, éstos se calientan y en medio acuoso se hinchan absorbiendo agua gracias a la gran cantidad de grupos hidroxilo que poseen. El agua eleva su energía cinética y penetra el grano de almidón que pierde birrefringencia y se inicia el espesamiento de la mezcla. Este proceso de empastamiento es por etapas y el incremento final de la espesura durante el calentamiento es debido al exudado de los granos hinchados que pueden plegarse y permanecer suspendidos en el liquido (Charley H., 1987). Los granos de almidón de papa cocidos son elásticos y muy deformables, lo que confiere caracteristicas de cohesividad y extensibilidad.

Cuando el grado de desparramamiento se hace menor $(5,7 \mathrm{~cm})$ por efecto de un sobrecalentamiento, el producto frío es rígido ya que al enfriar la energía potencial no es tan grande para contrarrestar la tendencia de la amilosa a reasociarse, la que se fija a otras moles de amilosa y al almidón en los extremos externos de los gránulos, formando una red. Los almidones que poseen amilosa de tamaño intermedio tienen la capacidad de inmovilizar liquido, que se une a las moléculas de almidón de la superficie de los granos hinchados y a la amilosa que enlaza a dichos granos. Por otro lado, el azúcar limita el hinchamiento de los granos de almidón por competir con ellos por el agua y los hace más resistentes a la ruptura mecánica. 
DIAGRAMA N ${ }^{\circ}$ 02: Variación de la temperatura y el grado de desparramamiento en el proceso artesanal.

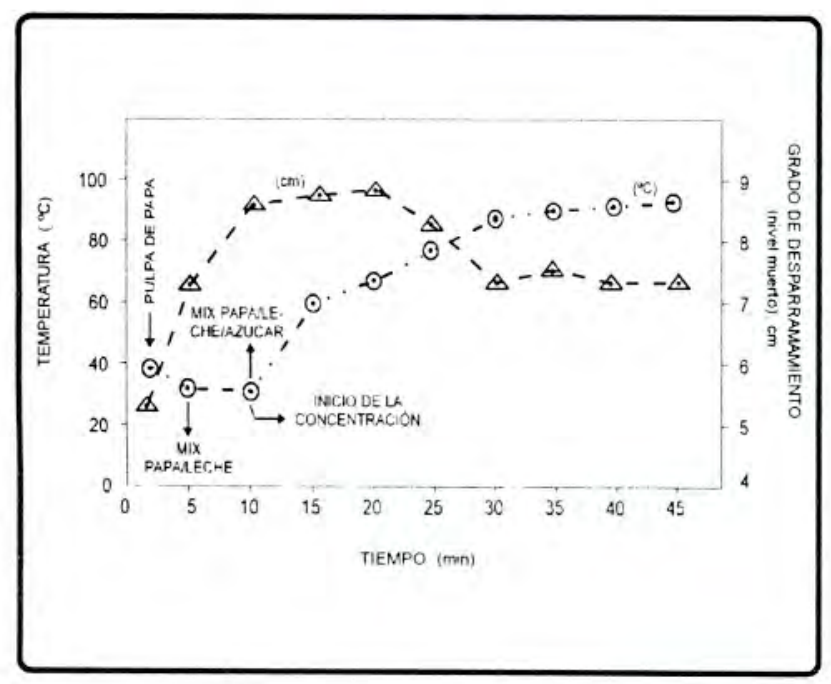

El empastamiento del almidón es afectado significativamente por la concentración de sacarosa y lactosa. La adición de sólidos de leche también regulan el contenido de sacarosa, especialmente la materia grasa.

Respecto al color que va adquiriendo la mezcla, se puede decir que de un tono amarillo intenso de la pulpa de papa pasa a un color amarillo claro por incorporación de la leche, que se oscurece al añadir azúcar (temperatura de la mezcla, $30^{\circ} \mathrm{C}$ ). El dulce de papa al final de la evaporación presenta color amarillomarrón claro; y es conocido que el efecto que ejerce el calor sobre los azúcares está relacionado con reacciones que producen pigmentos en los alimentos, a $\mathrm{pH}$ tanto ácido como alcalino. Por la composición de la mezcla a concentrarse una de estas reacciones sería la reacción de caramelización o de Maillard (Cheftel J, y Cheftel H, 1976), responsable de la presencia de melanoidinas.

Los sustratos presentes serían los carbonilos, grupos aminos y vitaminas como la $\mathrm{C}$.

Los azúcares reductores actuantes en este caso: glucosa, fructosa y lactosa se consideran medianamente reactivos, siendo la temperatura de trabajo un catalizador muy eficaz. Con respecto al $\mathrm{pH}$, su efecto es muy complejo ya que cada reacción tiene su pH óptimo (entre 6 y 8 para la condensación de Maillard, cerca a 7 para la reestructuración de Amadori, 5,5 para degradación de cetosaminas y $\mathrm{pH}$ aún menor para la degradación aerobia y anaerobia del ácido ascórbico). En lo que respecta al dulce de papa amarilla cuyo $\mathrm{pH}$ es de $6,44\left(\right.$ a $23,9^{\circ} \mathrm{C}$ ), se le puede clasificar como un alimento con condiciones favorables para la reacción de Maillard.

\subsection{VARIACIÓN DEL FLUJO ARTESANAL:}

Manteniendo constante la proporción papa/ azúcar/leche se varió el flujo de producción de acuerdo al siguiente esquema:

DIAGRAMA N ${ }^{\circ}$ 03: Flujo tentativo para la producción de dulce de papa.

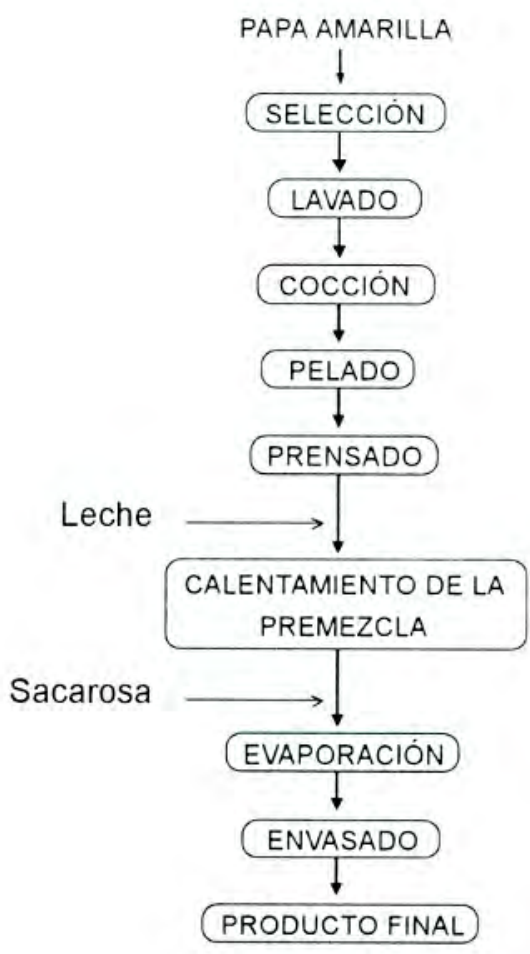

Durante el proceso se tomó mediciones de la temperatura de trabajo, consistencia de la mezcla (grado de desparramamiento, medido en $\mathrm{cm}$ ), el tiempo que demora cada operación y el color del producto. Los resultados obtenidos se muestran en el siguiente diagrama. 
DIAGRAMA N ${ }^{\circ}$ 04: Variación de la temperatura y el grado de desparramamiento (flujo de proceso tentativo).

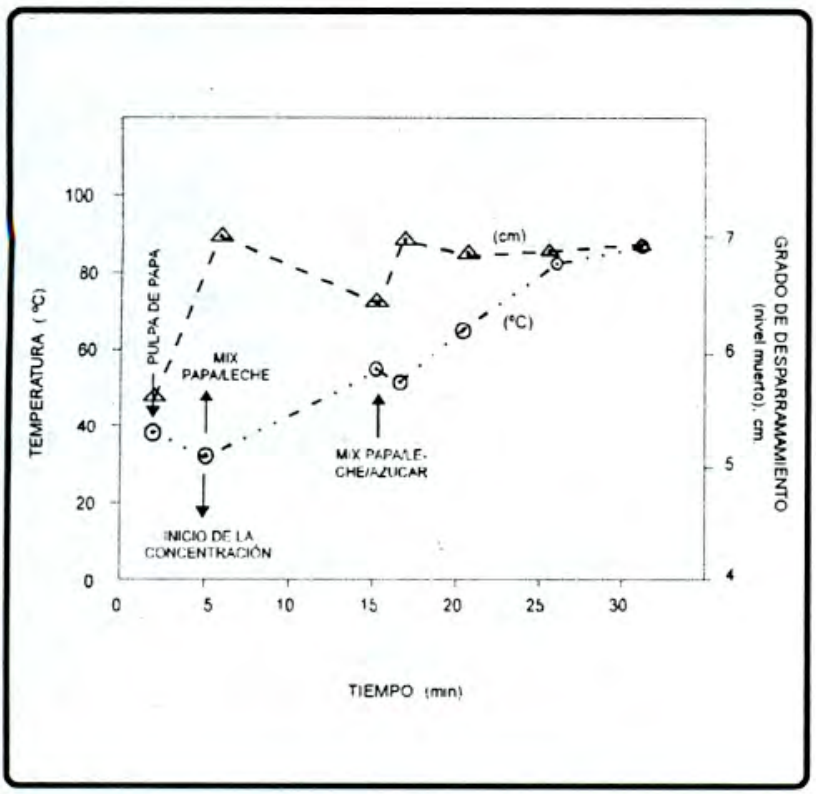

La secuencia de operaciones empleada redujo el tiempo de concentración por evaporación, la consistencia fue menos variable que en el flujo artesanal; de igual manera el color de la mezcla y del producto final se oscureció en menor grado, teniendo el dulce de papa amarilla color amarillo oscuro al final del proceso, evitándose el pardeamiento excesivo del producto. Además, el rendimiento final se incrementó a $72 \%$, muy superior al que se obtuvo en el dulce de leche con $0,2 \%$ de glucosa y $0,5 \%$ de almidón de maiz (43\%, según Chacón 1978), observándose que la adición de la leche, al inicio de la concentración, permite la absorción del agua en forma rápida permaneciendo el color inalterable. La adición del azúcar a $56^{\circ} \mathrm{C}$, además de conferir el sabor dulce característico, regula la consistencia final ( $7 \mathrm{~cm}$ para el grado de desparramamiento a $\left.90^{\circ} \mathrm{C}\right)$.

\subsection{FLUJO DEFINITIVO}

De acuerdo a los resultados obtenidos, el flujo artesanal puede ser variado con considerables ventajas de mayor rendimiento, protección de la calidad nutricional del alimento, y menor consumo de combustible y tiempo de trabajo.
DIAGRAMA N ${ }^{\circ} 05$ : Flujo propuesto para la producción de dulce de papa amarilla.

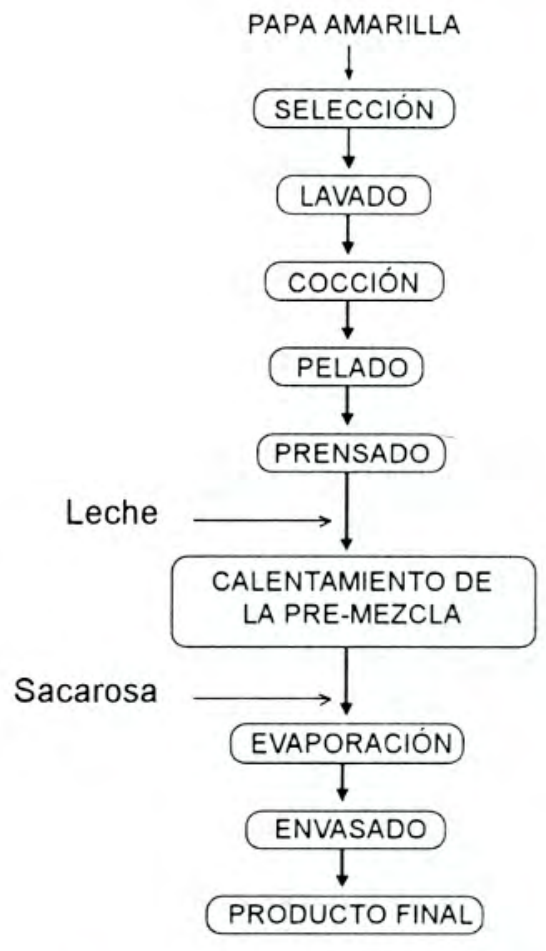

En el flujo de procesamiento se deberá considerar los siguientes aspectos:

- Selección de la papa amarilla, preferentemente de tamaño uniforme, sana y no germinada.

- Cocción completa (de allí la importancia de la selección de la materia prima).

- Pelado eficiente, cuidando de retirar los ojos para que no pasen a la pulpa.

- Prensado o pulpeado muy fino.

- Calentamiento con agitación posterior a la incorporación de la leche evaporada (hasta $55^{\circ} \mathrm{C}$ ), y adición de azúcar.

- Evaporación y evaluación de la conśistencia hasta el final de la operación.

- Envasado en materiales adecuados (PE de alta densidad, vidrio, PET, etc.).

\subsection{SUSTITUCIÓN PARCIAL DE LA LECHE EVAPORADA POR LECHE EN POLVO RECONSTITUIDA}

Con la finalidad de conocer la posible influencia del uso de leche entera en polvo en la 
formulación, se sustituyó la leche evaporada entera por leche en polvo reconstituida a doble concentración. El flujo de procesamiento fue el desarrollado en el Item 3.4 con las siguientes proporciones y códigos muestrales.

\begin{tabular}{|c|c|}
\hline \% DE SUSTITUCIÓN CON & CODIGO DE LA MUESTRA \\
LECHE ENTERA EN POLVO & B \\
\hline 0 & 0 \\
25 & $\mathrm{M}$ \\
50 & $\mathrm{~T}$ \\
75 & $\mathrm{R}$ \\
100 & \\
\hline
\end{tabular}

Las muestras obtenidas se analizaron sensorialmente utilizando un panel semientrenado constituido por 15 personas, y el test de ordenamiento por el que los panelistas calificaron las muestras colocando en primer lugar la que más les agradaba y en último lugar la menos agradable. Los resultados se presentan en el siguiente diagrama.

DIAGRAMA N06: Test de ordenamiento.

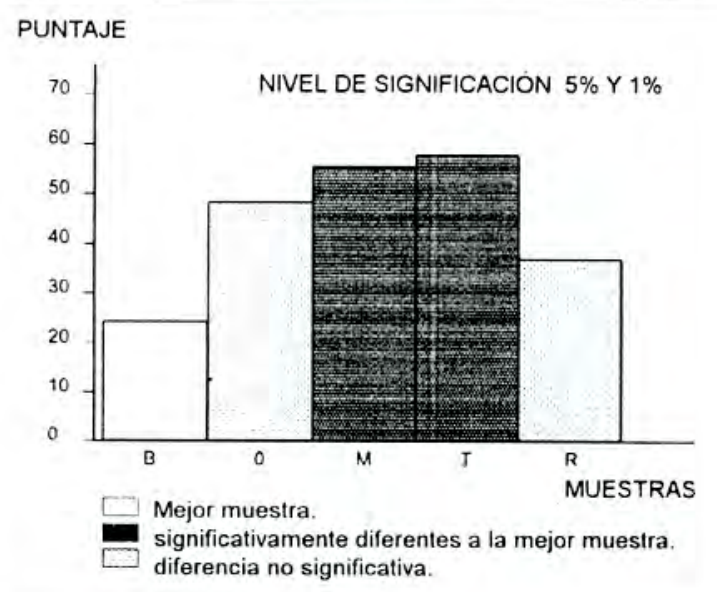

En las tablas correspondientes para un nivel de significación del 5\% (32-58) los resultados de las muestras B y $T$ son estadisticamente significativas, considerándose como la mejor la $B$, mientras que la $T$ fue rechazada. Para un nivel de significación del 1\% (30-60) también la muestra $B$ posee un resultado estadisticamente significativo y se le considera como la mejor muestra; existiendo un $95 \%$ de seguridad de que el rechazo de la prueba $T$ no es debida al azar. En este caso la menor diferencia significativa es $58-32=26$ para $5 \%$ y $60-30=30$ para $1 \%$ y todos los tratamientos con un ranking total sobre $50(26+24)$ son significativamente diferentes al tratamiento $\mathrm{B}$ (muestras $\mathrm{M}$ y $\mathrm{T}$ al $5 \%$ ), mientras que los tratamientos con un ranking total sobre $54(30+24)$ son significativamente diferentes al tratamiento $B$ (muestras $\mathrm{M}$ y T al $1 \%$ ).

\subsection{DEFECTOS QUE PUEDEN PRODUCIRSE EN EL DULCE DE PAPA AMARILLA}

Los defectos observados pueden deberse a las caracteristicas de la papa amarilla empleada, el proceso o a la presencia de microorganismos contaminantes.

a) Presencia de grumos pequeños de papa, cuando el pulpeado no es suficientemente fino.

b) Desarrollo de hongos y bacterias contaminantes, por falta de higiene en el procesamiento o envasado.

c) Color muy oscuro, si se acumulan excesivamente los azúcares reductores (glucosa, lactosa y fructuosa) afectándose también el sabor, comportamiento en la cocción y color, ya que estos azúcares contribuyen al pardeamiento no enzimático.

d) Sinéresis, debida al exceso de humedad en el producto final o a la acidez muy elevada.

e) Falta de consistencia, por incorporación de ácido que fragmenta los gránulos hinchados catalizando el calor la hidrólisis del almidón a dextrinas.

f) Dulce cuarteado, cuando se separan las fases y se produce Sinéresis. Este defecto va acompañado con la acidez excesiva.

\subsection{COMPOSICIÓN PROXIMAL DE LA FORMULACIÓN DEFINITIVA}

El dulce de papa producido con el flujo definitivo presentó las características que se citan a continuación:

CUADRO $\mathrm{N}^{\circ}$ 05: Composición proximal promedio del dulce de papa amarilla.

\begin{tabular}{|l|c|}
\hline CONSTITUYENTE & PORCENTAJE \\
\hline Humedad & 35,46 \\
Proteina & 5,10 \\
Ceniza & 1,090 \\
Fibra & 0,02 \\
Azúcares reductores & 27,93 \\
pH $\left(23,9^{\circ} \mathrm{C}\right)$ & 6,44 \\
Densidad $\left(20^{\circ} \mathrm{C}\right)$ & $0,113 \mathrm{gr} / \mathrm{ml}$ \\
Grado de desparramamiento $\left(90^{\circ} \mathrm{C}\right)$ & $7,0 \mathrm{~cm}$ \\
\hline
\end{tabular}

En el cuadro podemos observar que el 
contenido de humedad es superior al valor recomendado por IRAM (1971) para dulce de leche $(30 \%)$, por la incorporación de almidones provenientes de la papa que permiten mayor rendimiento. El contenido de ceniza y azúcares reductores están dentro de los estándares dados por el mismo autor (máximo $2 \%$ y $56 \%$ respectivamente), no asi el porcentaje de proteína presente (de 5,10\% para el dulce de papa amarilla y de $6 \%$ para el dulce de leche), ya que la papa amarilla aporta menos proteinas a la formulación $(2,18 \%)$. El pH del producto final fue de 6,44 , ligeramente menor que el dulce de leche comercial $\left(6,64\right.$ a $\left.23,9^{\circ} \mathrm{C}\right)$, este valor es resultado de la proporción papa amarilla/azúcar/leche evaporada utilizada sin adición de ácido.

La densidad del producto final $(0,113 \mathrm{gr} / \mathrm{ml})$ está relacionada con el grado de desparramamiento del producto $(7,0 \mathrm{~cm})$ al final del proceso; porque confiere al producto caracteristicas sensoriales adecuadas como son la facilidad para untarse, buena consistencia, color amarillo-marrón claro, sabor agradable y dulce, ligero olor a papa y apariencia general apetecible. Es importante señalar la ausencia de cristales de tamaño mayor a 25 micras que generalmente causan arenosidad en el dulce de leche y constituyen un problema que afecta la calidad de ese producto, lo que se comprobó durante la conservación del dulce de papa a $12^{\circ} \mathrm{C}$ por dos meses. La densidad final es menor que la conseguida para el dulce de leche con la adición de $0,5 \%$ de almidón de maíz $(0,1429 \mathrm{gm} / \mathrm{ml})$, (Chacón H., 1978).

Tanto las características físicas y químicas como las sensoriales se mantuvieron en el producto almacenado en bolsas de polietileno de doble densidad, a $12^{\circ} \mathrm{C}$ durante dos meses.

En envases rígidos de PET se observa que la densidad varía ligeramente por evaporación de

ANTUNEZ DE MAYOLO S., 1988. La alimentación en el antiguo Perú. Ed. del Banco Central de Reserva. Lima - Perú.

BADUI S., 1981. Química de los alimentos. Ed. Alhambra, Méjico. CENTRO INTERNACIONAL DE LA PAPA (CIP). Reporte de papa amarilla del Centro, Runtun (Solanum goniocalyx).

COLLAZOS C. et al, 1993. La composición de alimentos de mayor consumo en el Perú. Inst. de Nutrición, $6^{\circ}$ Ed. Lima.

CHACON H., 1978. Utilización de la glucosa, almidón de maiz y lactosa en la elaboración de manjarblanco (dulce de leche). Tesis UNALM, Lima-Perú.

CHARLEYH., 1987. Tecnologia de alimentos. Ed. Limusa. Méjico. CHEFTEL J., CUQ J., y LORIENT D., 1989. Proteinas alimentarias. agua contenida en el dulce de papa $(0,114 \mathrm{gr} / \mathrm{ml})$.

\subsection{PRUEBA DE ALMACENAJE $\left(12^{\circ} \mathrm{C}\right.$ POR 60 DIAS)}

Se almacenaron muestras correspondientes a la formulación óptima por dos meses a temperatura de $12^{\circ} \mathrm{C}$, que de acuerdo a investigaciones realizadas por Hosken (1969; citado por Chacón, 1978), es una temperatura comprendida en el rango óptimo para almacenar dulce de leche.

Los métodos microbiológicos empleados fueron los de Mossel y Quevedo (1967) y de acuerdo a la Norma Argentina para evaluación microbiológica de dulce de leche y de González, (Chacón H. 1978); fueron analizadas la presencia de microorganismos patógenos, hallándose staphylococcus aureus coagulasa negativa y Klebsiella, enterobacteria que puede provenir de contaminación por falta de higiene.

Ambos organismos estuvieron presentes en un número menor de 10 colonias/gr. La evaluación sobre presencia de hongos y levaduras dio resultado negativo.

Estos resultados indican que es posible mantener el producto en condiciones sanitarias adecuadas, durante dos meses y almacenadas a $12^{\circ} \mathrm{C}$, sin uso de conservantes.

\section{CONCLUSIÓN}

De acuerdo a los resultados de las terminaciones químicas y microbiológicas evaluadas, el dulce de papa amarilla obtenido presenta caracteristicas estables y muy adecuadas desde el punto de vista alimenticio, siendo ampliamente aceptado por sus cualidades sensoriales. Asimismo, el rendimiento, de por si bueno, se pudo elevar mejorando el flujo de procesamiento hasta el $72 \%$, reduciéndose el tiempo de trabajo y uso de combustible para ofrecer mayor perspectiva industrial.

\section{REFERENCIAS BIBLIOGRÁFICAS}

Ed. Acribia, España

IRAM. 1971. Dulce de leche Instituto de racionalización de materiales. Argentina.

ITINTEC. 1975. Norma N² 207.001. Azúcar de caña. Lima-Perú.

MOSSEL D., Y QUEVEDO F., 1968. Control microbiológico de los alimentos. Monografía Cleiba. Lima-Perú.

MOSSEL D., y MORENO B., 1982. Microbiologia de los alimentos. Ed. Acribia S.A. España.

PEARSON D., 1986. Técnicas de laboratorio para el análisis de alimentos. Ed. Acribia. España. 